\title{
Research Paper: Evaluation of the Effect of Vestibular Exercises on Dizziness and Postural Control in Parkinson Patients
}

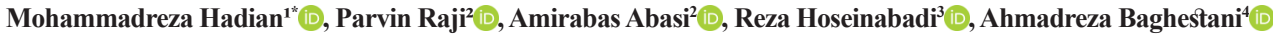 \\ 1. Brain and Spinal Cord Injury Research Center, Neuroscience Institute, International Campus, Tehran University of Medical Sciences, Tehran, Iran \\ 2. Department of Occupational Therapy, School of Rehabilitation, Tehran University of Medical Sciences, Tehran, Iran. \\ 3. Department of Audiology, School of Rehabilitation, Tehran University of Medical Sciences, Tehran, Iran. \\ 4. Department of Biostatistics, School of Allied Medical Sciences, Shahid Beheshti University of Medical Sciences, Tehran, Iran.
}

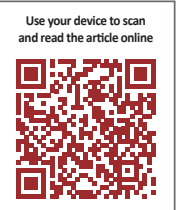

Citation: Hadian M, Raji P, Abasi A, Hoseinabadi R, Baghestani A. Evaluation of the Effect of Vestibular Exercises on Dizziness and Postural Control in Parkinson Patients. Journal of Modern Rehabilitation. 2018; 12(1):13-20. http://dx.doi. org/10.32598/jmr.12.1.13

http://dx.doi.org/10.32598/jmr.12.1.13

Funding: See Page 19

(c) Copyright: The Author (s)

Article info:

Received: 17 Aug 2017

Accepted: 25 Nov 2017

Available Online: 01 Jan 2018

\section{Keywords:}

Parkinson disease, Dizziness, Vestibular exercises, Physical rehabilitation

\begin{abstract}
Introduction: Non-motor symptoms of Parkinson disease have significant effects on the quality of life in this group of patients. Among these symptoms, dizziness is associated with the changes in Orthostatic Hypotension $(\mathrm{OH})$. About $30 \%$ of people over 65 years have dizziness; However, the exact mechanism of dizziness in these patients was not clear. Dizziness has symptoms such as fainting, light headache, vertigo and imbalance. The present study is based on the given fact that one of the reasons for dizziness in Parkinson patients is the disturbance of balance systems. In addition, the vestibular system is one of the main elements involved in normal balance. As, the role of vestibular system in Parkinson disease has been suggested in previous studies. The main aim of current study is to investigate the effects of vestibular exercises on the dizziness of Parkinson patients.
\end{abstract}

Materials and Methods: Twenty-four patients participated in this study based on the inclusion criteria and were randomly assigned into intervention and control groups. Dizziness Handicap Inventory-Persian (DHI-P) was used for dizziness measurement. In addition, Berg Balance Scale (BBS), Functional Reach (FR) and 2 Minutes Walking Test (2MWT) were used for measuring the postural control before and after interventions. The intervention group performed the vestibular exercises and the control group performed the conventional exercises (3 days a week for 60 minutes and a total of 24 sessions).

Results: The total score of DHI-P showed a significant improvement in the intervention group compared to the control group ( $\mathrm{P}<0.001)$. Also, the BBS tests $(\mathrm{P}<0.001)$, FR $(\mathrm{P}<0.001), 2 \mathrm{MWT}$ $(\mathrm{P}=0.001)$ showed a significant improvement in the intervention group.

Conclusion: Based on the results of this study, it could be suggested that vestibular exercises, as none sophisticated, feasible, and low cost rehabilitation-protocol has beneficial effects for patients with Parkinson disease. This protocol does not need any particular equipment and can be used in all environments while it can reduce dizziness and improve motor skills and postural control in this group of patients.

* Corresponding Author: 


\section{Introduction}

$\mathbf{P}$

arkinson disease is among the progressive diseases of nervous system. The pathology of Parkinson disease is attributed to the impairment of basal ganglia and the disease is diagnosed with symptoms such as motor retardation, joints stiffness, tremors and postural instability [1]. According to a study, the rate of Parkinson disease was estimated to be 222.9 patients per 100000 people in Tehran [2].

Vestibular dysfunction is associated with Postural and equilibrium disturbances, and a number of problems such as imbalance and persistent falls, immobility and bed ridden are the consequences of these common symptoms [3]. Ample of evidences had shown the role of sensory signals and in particular, sensory integrity from various organs such as mechanoreceptors, vestibular system and special sense (i.e. vision) for normal equilibrium and balance. This means that the sensory messages from vestibular organs together with the visual sensory messages and signal from proprioceptors are being integrated with the activity of the cerebellum, extra pyramidal and limbic systems and cortex [4]. In case of disturbances of these systems, they might cause postural instability, dizziness, and vertigo [5].

Dizziness is one of the Non-motor symptoms of this disease which have significant impact on the quality of life of the Parkinson patients. Dizziness is attributed to the changes in Orthostatic Hypotension $(\mathrm{OH})[6]$; Although, about $30 \%$ of people over 65 years are affected by dizziness, but the precise mechanism is not fully understood in Parkinson patients [7]. Dizziness has symptoms such as fainting, light headaches, vertigo and imbalance [8].

The aim of this study was to investigate the role of vestibular system as one of the important organs involved in balance by considering disturbances in orientation, equilibrium and sensory integrity systems as sources of dizziness in Parkinson patients. Based on the previous studies, Parkinson patients suffered from vestibular dysfunction [3] and it could be managed by vestibular rehabilitation exercises [9]. Vestibular rehabilitation is a group of exercises for patients with balance and equilibrium disorders; these exercises can stimulate the plasticity of central nervous system and accordingly, improve the postural stability. Furthermore, they teach the brain how to adapt to unspecified stimuli [3].
As it was suggested, vestibular dysfunction has devastating effects on the daily activities of patients [10] Awareness of the negative effects of vestibular dysfunction on quality of life is an important part of the Vestibular rehabilitation program. In this study, the translated version of DHI [11] was used; DHI (Dizziness Handicap Inventory) is the first self-assessment questionnaire to assess the disability caused by dizziness and also to evaluate the effects of vestibular exercises on rehabilitation plans. [12].

\section{Materials and Methods}

This is an interventional study with a single-blind clinical trial design. Evaluations were made by another specialist who was not aware of the patients grouping. Sampling was based on the availability; all patients who entered to the current study were among the Parkinson patients referring to Rasoul-e-Akram and Imam Khomeini hospitals. In first stage, five subjects participated in the study as a pilot sample and then; the sample size were determined based statistical analysis and then, 24 patients were voluntarily participated in the study. Twelve subjects were then randomly assigned in to the control group (6 women, 6 men, mean age 63.08 yrs.) and 12 subjects to the intervention group ( 8 men, 4 women, mean age 63.16 yrs.) based on the inclusion criteria through encoded envelopes.

The inclusion criteria for the study were as following: Disease level of 1 to 4 according to the Hoehn and Yahr ( $\mathrm{H} \& \mathrm{Y}$ ) scale [3], the ability to walk with or without axillary crutches for at least 100 meters [13], videonystagmography indicating the Vestibular disorders, performed by an Audiologist [14], cognitive performance above 23 on the MMSE test [15], depression score less than 29 based on Beck Depression Inventory (BDI) [16, 17], lack of severe sleep disorder based on the Pittsburgh Sleep Quality Index (PSQI-P), score less than 5 [18] and lack of stress and anxiety based on Depression Anxiety Stress Scales-21 (DASS-21) [19].

Depression, anxiety and stress item have a score of less than 20,14 and 25 respectively [20]. All tests and evaluations were performed in condition that the symptom of the disease related to non-use of the medications drug had completely subsided [21]. However, the medication consumption was discontinued 24 hours before the videonystangography test [14]. No diabetes, no pain related to osteoarthritis and osteoporosis, particularly in lower limbs, no neurological diseases including stroke or severe visual and auditory disorders. The exclusion criteria 
for the study were lack of cooperation during the tests and exercises.

\section{Protocol}

The patients were asked to complete the Dizziness Handicap Inventory-Persian (DHI-P) questionnaire after completing the consent form; then the assessments were performed by an occupational therapist who was not aware of grouping. The patients were randomly assigned in to the intervention and control groups. In the intervention group, Vestibular exercises, as described in appendix [13], were completely performed for 24 sessions about 55 minutes to 60 minutes, 3 days a week. The patients were also allowed to rest for 1 minute in the event of a feeling tired or to resume another appointment if they were unable to continue the workout.

In the control group, conventional exercises included 5 minutes of warm-up (slow and inactive walking), muscle stretching exercises (scapular muscles, pelvic flexors, hamstrings and gastrocnemius) [22] and also standing and sitting body rotation were performed for 15 minutes respectively. The patients were given time to rest during the training if they felt tired. To assess the effectiveness of Vestibular Rehabilitation interventions, Berg Balance Scale (BBS), Functional Reach (FR) and 2 Minutes Walking Test (2MWT) were performed before and after the interventions.

Dizziness with Dirty Handicap Inventory-Persian (DHI-P) questionnaire consists of 25 questions, divided into three sub-items: 9 physical questions, 9 functional questions and 7 emotional questions. There are 3 options for each question: Yes with 4 points (highest score), sometimes with 2 points and no with 0 points. The total score of this questionnaire is between 0-100, in a way that 100 indicates the highest level of dizziness. The score below 30 represents mild disabilities, 31-60 reports moderate disabilities and more than 60 indicates severe disabilities. Nineteen is the minimum score for discriminating variations in DHI-P [11]. Also, Cronbach's alpha for DHI-P, the emotional sub item, the physical sub item and the functional sub-item were 0.79 , $0.82,0.83$ and 0.9 for total; respectively.

Based on the reliability and good internal correlation found in the Persian version of DHI-P, it provided a reliable tool for assessing the effect of dizziness on quality of life [11]. Berg Balance Scale (BBS), as a basic functional test consists of 14 items which is, scored from 0 to 4 . Total score of this scale is 56 that represents excellent balance and a score of 0 indicates severe equilib- rium damage. The Persian version of $\mathrm{BBS}$ is also a valid and reliable instrument for Iranian elderly and Cerebral Palsy children (ICC $=0.93,0.094$ respectively) $[23,24]$.

Functional Reach test (FR) has been developed as a rapid screening tool for balance problems and risk of falling in elder population. To perform this test, the subject stands up, opens the legs as wide as the width of the shoulders and lifts and stretches the hand for 90 degrees from the front. The subject stands in stable position, and extend his hand forward as long as he can while, maintaining his balance; then, the distance of the stretched hand is measured by a meter; this test has a test-retest reliability $(\mathrm{ICC}=0.94)$ [25].

There are 12 minute walking, 6 minutes walking and 2 minutes walking tests for tolerance evaluation and there are no differences between these tests in the evaluation value of tolerance. However, the 2 minutes walking test is easier and less time consuming for the patients. Therefore, this test was used in this study [26]. To carry out these tests, the subject stands behind a starting line marked on the ground and the therapist asks him to walk as quick as possible a 21 meters distance; as therapist set the chronometer for 2 minutes and the distance will be measured at the end [27].

\section{Statistical analysis}

The variables (DHI-P, total score and sub items, Berg, FR and 2MWT were compared by independent sample $t$ test for both groups and $\mathrm{P}<0.05$ was considered statistically significant (SPSS V.24). The paired t test was used (based on normality of data) for comparison of the scores before and after the intervention in both groups. Also, to determine the significance level of information about the inclusion criteria between the two groups, including demographic data such as age, gender and history of the disease, the two independent t-test and chisquare tests were used.

\section{Results}

Twenty four Parkinson patients were evaluated and treated based on the inclusion criteria in this study. They were randomly assigned in two groups. The demographic information and entry criteria were shown in Table 1; Normal distribution of data based on kolmogrov-smirnov test was shown and no significant differences were between the groups $(\mathrm{P}=0.352)$ Twelve subjects were in the vestibular exercise group (mean age 16.63), of which 4 were afflicted with grade III and 8 subjects had grade II Parkinson according to Hoehn \& 
Table 1. Demographic data, mean scores and standard deviation

\begin{tabular}{cccc}
\hline Variable & Intervention Group & Control Group & Significance Level \\
\hline Age (year) & $63.16(\mathrm{SD} \pm 8.05)$ & $63.08(\mathrm{SD} \pm 9.49)$ & 0.982 \\
\hline Gender (male/ female) & 4.8 & 6.6 & $0.408^{\mathrm{a}}$ \\
\hline History of disease (year) & $3.33(\mathrm{SD} \pm 1.55)$ & $3.75(\mathrm{SD} \pm 1.60)$ & 0.525 \\
\hline H \& Y Grade II/ III & 4.8 & 5.7 & $0.178^{\mathrm{a}}$ \\
\hline BMI & $25.30(\mathrm{SD} \pm 3.18)$ & $25.07(\mathrm{SD} \pm 4.19)$ & 0.884 \\
\hline MMSE & $24.81(\mathrm{SD} \pm 0.98)$ & $25.23(\mathrm{SD} \pm 1.01)$ & 0.549 \\
\hline
\end{tabular}

Normal of distribution of data in both intervention and control groups based on Kolmogrov-Smirnov test.

a: $P$ was assessed by K square test

Table 2. Dizziness scores within the groups before and after the intervention (at the end of $24^{\text {th }}$ session)

\begin{tabular}{|c|c|c|c|}
\hline & Variable & Control & Intervention \\
\hline \multirow{5}{*}{$\begin{array}{l}\text { DHI physical sub } \\
\text { item }\end{array}$} & Before the intervention & $19.50(\mathrm{SD} \pm 8.27)$ & 18.33(SD \pm 7.32$)$ \\
\hline & After the intervention & $19.33(\mathrm{SD} \pm 8.05)$ & $16(\mathrm{SD} \pm 5.72)$ \\
\hline & Changes & $-0.16(S D \pm 1.33)$ & $-2.33(\mathrm{SD} \pm 2.93)$ \\
\hline & $95 \% \mathrm{Cl}$ & -1.01 to 0.68 & -4.19 to -0.46 \\
\hline & $P$ & 0.674 & 0.019 \\
\hline \multirow{5}{*}{$\begin{array}{l}\text { DHI emotional sub } \\
\text { item }\end{array}$} & Before the intervention & $10.66(S D \pm 5.86)$ & $13.50(\mathrm{SD} \pm 7.77)$ \\
\hline & After the intervention & $10.66(S D \pm 5.86)$ & $11.16(\mathrm{SD} \pm 5.62)$ \\
\hline & Changes & 0 & $-2.33(\mathrm{SD} \pm 3.49)$ \\
\hline & $95 \% \mathrm{Cl}$ & 0 & -4.55 to -0.46 \\
\hline & $P$ & - & 0.041 \\
\hline \multirow{5}{*}{$\begin{array}{l}\text { DHI functional sub } \\
\text { item }\end{array}$} & Before the intervention & $13.83(\mathrm{SD} \pm 7.15)$ & $19.33(\mathrm{SD} \pm 7.78)$ \\
\hline & After the intervention & $13.83(\mathrm{SD} \pm 7.15)$ & $12.66(\mathrm{SD} \pm 5.54)$ \\
\hline & Changes & 0 & -6.66 \\
\hline & $95 \% \mathrm{Cl}$ & 0 & -9.33 to -3.99 \\
\hline & $P$ & - & $<0.001$ \\
\hline \multirow{5}{*}{ DHI total score } & Before intervention & $44(S D \pm 18.15)$ & $51.16(S D \pm 18.78)$ \\
\hline & After the intervention & $43.83(S D \pm 17.99)$ & $39.83(S D \pm 13.84)$ \\
\hline & Changes & $-0.16(S D \pm 1.33)$ & $-11.33(S D \pm 6.16)$ \\
\hline & $95 \% \mathrm{Cl}$ & -1.01 to 0.68 & -15.25 to -7.41 \\
\hline & P & 0.674 & $<0.001$ \\
\hline
\end{tabular}


Table 3. BBS, FR, 2MWT test scores within the groups before and after intervention (end of session 24)

\begin{tabular}{|c|c|c|c|}
\hline Variable & Variable & Control Group (n=12) & Intervention Group ( $n=12$ ) \\
\hline \multirow{5}{*}{ BBS } & Before the intervention & $45.91(S D \pm 6.18)$ & $47.83(S D \pm 4.78)$ \\
\hline & After the intervention & $46.08(S D \pm 6.03)$ & $55(S D \pm 14)$ \\
\hline & Changes & $0.16(S D \pm 0.57)$ & $7.16(S D \pm 3.24)$ \\
\hline & $95 \% \mathrm{Cl}$ & -0.20 to 0.53 & 5.10 to 9.22 \\
\hline & $P$ & 0.339 & $<0.001$ \\
\hline \multirow{5}{*}{$F R$} & Before the intervention & $21.83(S D \pm 5.84)$ & $21.41(S D \pm 4.88)$ \\
\hline & After the intervention & $22.91(S D \pm 5.4)$ & $28.41(S D \pm 4.20)$ \\
\hline & Changes & 1.08 & $7(2.17)$ \\
\hline & $95 \% \mathrm{Cl}$ & 0.36 to 2.52 & 5.61 to 8.38 \\
\hline & $P$ & 0.127 & $<0.001$ \\
\hline \multirow{5}{*}{ 2MWT } & Before the intervention & $113.75(\mathrm{SD} \pm 28.12)$ & $116(S D \pm 24.84)$ \\
\hline & After the intervention & $115(S D \pm 29.01)$ & $139.75(S D \pm 24.30)$ \\
\hline & Changes & 1.25 & 23.75(SD \pm 16.73$)$ \\
\hline & $95 \% \mathrm{Cl}$ & 0.26 to 2.76 & 13.11 to 34.38 \\
\hline & P & 0.096 & 0.004 \\
\hline
\end{tabular}

95\%Confidence Interval (95\% CI): 95\% confidence interval and P; (P) Showed significance level before and after the intervention for both groups.

Yahr scale. In addition, 12 subjects were in conventional exercise group (mean age $63.08 \mathrm{yrs}$ ), of which 5 were afflicted with grade III and 7 others had grade II of the disease. In the control and intervention groups, the mean total scores of DHI-P, before the intervention were 44 $(\mathrm{SD}=18.15)$ and $16.51(\mathrm{SD}=18.78)$ respectively, The total score of DHI-P and the sub items before and after the intervention in the control and intervention groups were presented in Table 2.

The findings showed that the total score of DHI-P ,after training, in the intervention group was lower than the control group. In the intervention group, 4 patients (33\%) reported an improvement of more than 19 points compared to before intervention in DHI-P. However, no (i.e. 19 points) changes were observed in the control group. The scores of BBS, FR, 2MWT equilibrium tests were $45.91(\mathrm{SD}=6.18), 21.83(\mathrm{SD}=5.84)$ and $113.75(\mathrm{SD}=28.12)$ in the control group before intervention, respectively. The scores before intervention were 47.83(SD=4.78), 21.41(SD=4.88) and $116(\mathrm{SD}=24.84)$ respectively in the intervention group (Table 3 ). The scores of BBS, FR, 2MWT equilibrium tests showed a higher improvement in the intervention group compared to the control group after the intervention. The full report of the findings was presented in Tables 3, 4 .

\section{Discussion}

The results of present study showed that Vestibular exercises had significant effects on dizziness and improvement of postural control in Parkinson patients in comparison with the conventional exercises. The effectiveness of Vestibular exercises on dizziness and Improvements of Performances were also investigated by using scores of DHI-P, BBS and FR, and 2MWT. Based on our findings, there were significant improvements in the intervention group (i.e. vestibular rehabilitation) compared to the control group (conventional exercises).

Reviewing the literature, there were very few studies in this area for comparison of our data with previous research. In fact, the present study, by our knowledge, is one of the pioneers of clinical trial researches in this field reported some cliinical benefits of vestibular exercise for Parkinson patients; that is in line with our results. Zeigelboim also attributed their findings reinforced the hypothesis that "vestibular rehabilitation can 
Table 4. Comparison between intervention group and control in DHI, BBS, FR, 2MWT tests

\begin{tabular}{|c|c|c|}
\hline Variable & & Comparison Between the Intervention Group and the Control Group \\
\hline \multirow{3}{*}{ DHI physical sub item } & T & -2.328 \\
\hline & & \\
\hline & $\mathrm{P}$ & 0.034 \\
\hline \multirow[b]{2}{*}{ DHI emotional sub item } & $\mathrm{T}$ & -2.310 \\
\hline & P & 0.041 \\
\hline \multirow[b]{2}{*}{ DHI functional sub item } & $\mathrm{T}$ & -5.490 \\
\hline & P & $<0.001$ \\
\hline \multirow[b]{2}{*}{ DHI total score } & $\mathrm{T}$ & -6.128 \\
\hline & P & $<0.001$ \\
\hline \multirow{3}{*}{ BBS } & $\mathrm{T}$ & 6.860 \\
\hline & & \\
\hline & $\mathrm{P}$ & $<0.001$ \\
\hline \multirow[b]{2}{*}{ FR } & $\mathrm{T}$ & -6.514 \\
\hline & P & $<0.001$ \\
\hline \multirow[b]{2}{*}{ 2MWT } & T & -4.611 \\
\hline & $P$ & 0.001 \\
\hline
\end{tabular}

$\mathrm{T}$ an independent test and $\mathrm{P}$ showed a significant level before and after the intervention for both groups.

accelerate the mechanisms of central compensation by means of neuroplasticity with the execution of repetitive exercises" [9].

There is another study regarding the effects of virtual exercises on dizziness, albeit, the neurophysiological effects of virtual exercises might not be alike as vestibular exercises; however, it can be concluded that the significant difference between the DHI-P scores before and after Virtual exercises, reported by Severiano et.al, was in line with the finding of present research [28]. Furthermore, by comparing the results of our study with previous studies, one might argue that the differences between BBS $(\mathrm{P}=<0.001)$, FR $(\mathrm{P}=<0.001), 2 \mathrm{MWT} \quad(\mathrm{P}=0.004)$ scores could be attributed to the effects of Rehabilitation exercises that were compatible with the results of RossiIzquierdo et al. (2009), Hebert et al. (2011).

However, in the later study, Vestibular rehabilitation was used for multiple sclerosis $[3,13]$. In addition, previous study had suggested that part of the vestibular training protocol that was related to equilibrium exercises had conditioning effects; in other word, it might prepare the Central Nervous System (CNS) to provide efficient reac- tion. Consequently, these exercises could provide proper signals needed for the control of body during standing and walking [13]. Therefore, it seems that the significant results obtained in this study were somehow related to the improvement of motor and functional skills. Accordingly, As a result of decreasing dizziness, there was a positive effect on the physical and functional items of DHI questionnaire. For this reason, it might be suggested that Vestibular rehabilitation might cause central nervous system modulation in our patients.

Furthermore, in another study, Petzinger et al. suggested that neuroplasticity in Parkinson patients can be considered as a result of functional improvement. In other word, physical activity through exercises could cause some neuroplastic changes in brain, probably, at the levels of neurotransmitters, synaptogenesis and neurogenesis. This in turn, might cause, positive effects on neural circuits and, subsequent changes after training might occurs due to exercises [29]. Therefore, improvements of equilibrium and walking strategies partly are attributed to the muscle coordination after performing vestibular exercises. 
Previous studies had shown that saccade disorder and eye movement impairment were present in Parkinson patients based on videonystagmography results [14]. As, oculomotor exercises, is part of the vestibular exercises and comprise of saccades, pursuit (follow-up), and Vestibuloocular reflexes [13], consequently, oculomotor exercises might had some beneficial effects for the patients. Also, according to Oculomotor studies system, the Oculomotor disturbances could also be the representative of control disabilities Of these patients [30].

Oculomotor exercises could coordinate movements of the head, body, and pelvic girdle during walking. As a result, Oculomotor exercises in the form of eye movements might had a key role in neuromuscular reorganization through neuromuscular plasticity [31]. Hence, in other words, these exercises can maintain postural stability through Vestibulo-ocular and Vestibulo-spinal reflexes by activating the antigravity muscles and then, can enhance the functional abilities of the patients $[32,33]$.

The results of this study showed that Vestibular exercises can have significant Positive effects on dizziness, orientation and improve the equilibrium disorders and functional abilities of Parkinson patients. Reviewing of the relevant literatures, previous studies had attributed the positive changes to the neuroplasticity of central nervous system. It seems that relatively long term exercises, used in the current study, had been able to affect the mechanisms involved in neuroplasticity, although there is no direct evidence in this study for the measurement of changes in brain connections at molecular level and neural circuits.

Nevertheless, this might be inferred from the results of other studies that had shown evidences on the effect of training on neuroplasticity at the neurotransmitter levels, synaptogenesis, and neurogenesis [29]. With regard to the present evidences, we strongly suggest the vestibular exercises for the physical rehabilitation of Parkinson patients in order to improve dizziness, balance, daily activities and walking functions.

Limitations should be acknowledged. The objective tools that could be useful to evaluate, the progression of Dizziness and equilibrium, more efficiently and documentary such as Functional Magnetic Resonance Image (FMRI). Although the sample size should be more than this study to regard carefully ample of characters and differences between patients.

\section{Ethical Considerations}

\section{Compliance with ethical guidelines}

This study was under supervision of Ethics Committee of Research of Tehran University of Medical Science. The ethical code of this study was (IR.TUMS.FNM. REC.1396.3210).

\section{Funding}

This research was supported by Tehran University of Medical Sciences, School of Rehabilitation.

\section{Conflict of interest}

The authors declare no conflict of interest.

\section{Acknowledgements}

We are grateful for the sincere cooperation of patients who kindly participated in this study. Furthermore we are also sincerely grateful to our colleagues in Occupational and Physical Therapy Departments of Imam Hospital and Rasoul-e-Akram Hospital.

\section{References}

[1] Teive HA, Bertucci Filho DC, Munhoz RP. Unusual motor and non-motor symptoms and signs in the early stage of Parkinson's disease. Arquivos de Neuro-Psiquiatria. 2016; 74(10):781-4. [DOI:10.1590/0004-282X20160126] [PMID]

[2] Fereshtehnejad SM, Shafieesabet M, Rahmani A, Delbari A, Lökk J. Medium-to-high prevalence of screening-detected parkinsonism in the urban area of Tehran, Iran: Data from a community-based door-to-door study. Neuropsychiatric Disease and Treatment. 2015; 11:321-32. [DOI:10.2147/NDT.S77391] [PMID] [PMCID]

[3] Rossi-Izquierdo M, Soto-Varela A, Santos-Perez S, Sesar-Ignacio A, Labella-Caballero T, Rossi-Izquierdo M, et al. Vestibular rehabilitation with computerised dynamic posturography in patients with Parkinson's disease: Improving balance impairment. Disability and Rehabilitation. 2009; 31(23):1907-16. [DOI:10.1080/09638280902846384] [PMID]

[4] Luxon L. Evaluation and management of the dizzy patient. Journal of Neurology, Neurosurgery \& Psychiatry. 2004; 75(suppl 4):iv45-52. [DOI:10.1136/jnnp.2004.055285] [PMID] [PMCID]

[5] Angeli S, Marchese R, Abbruzzese G, Gandolfo C, Conti M, Gasparetto B, et al. Tilt-table test during transcranial Doppler monitoring in Parkinson's disease. Parkinsonism \& Related Disorders. 2003; 10(1):41-6. [DOI:10.1016/S1353-8020 (03)00069-5] 
[6] Goldstein D, Holmes C, Dendi R, Bruce S, Li S-T. Orthostatic hypotension from sympathetic denervation in Parkinson's disease Neurology. 2002; 58(8):1247-55. [DOI:10.1212/WNL.58.8.1247] [PMID]

[7] Yilmaz LÇ, Tunç T, İnan LE. The causes of dizziness in Parkinson's disease. International Journal of Clinical Medicine. 2014; 5(12):667-73. [DOI:10.4236/ijcm.2014.512092]

[8] Colledge N, Lewis S, Mead G, Sellar R, Wardlaw J, Wilson J. Magnetic resonance brain imaging in people with dizziness: A comparison with non-dizzy people. Journal of Neurology, Neurosurgery \& Psychiatry. 2002; 72(5):587-9. [DOI:10.1136/ jnnp.72.5.587] [PMCID]

[9] Zeigelboim BS, Klagenberg KF, Teive HA, Munhoz RP, Martins-Bassetto J. Vestibular rehabilitation: clinical benefits to patients with Parkinson's disease. Arquivos de Neuro-Psiquiatria. 2009; 67(2A):219-23. [DOI:10.1590/s0004282×2009000200009] [PMID]

[10] Santos JB, Garcia AP. [Study of the impact of the quality of life of individuals with dizziness (PortuguesE)]. Otolaryngology Act. 2007; 25(2):152-6.

[11] Jafarzadeh S, Bahrami E, Pourbakht A, Jalaie S, Daneshi A. Validity and reliability of the Persian version of the dizziness handicap inventory. Journal of Research in Medical Sciences. 2014; 19(8):769-75.

[12] Jacobson GP, Newman CW. The development of the dizziness handicap inventory. Archives of Otolaryngology-Head \& Neck Surgery. 1990; 116(4):424-7. [DOI:10.1001/archoh tol.1990.01870040046011]

[13] Hebert JR, Corboy JR, Manago MM, Schenkman M. Effects of vestibular rehabilitation on multiple sclerosis-related fatigue and upright postural control: A randomized controlled trial. Physical Therapy. 2011; 91(8):1166-83. [DOI:10.2522/ptj.20100399] [PMID]

[14] Hosseinabadi R, Sheibanizadeh A, Shahidi G, Gholamipur A, Kamali M. Survey of saccadic parameters using videonystagmography in patients with idiopathic Parkinson's disease and normal subjects. Audiology. 2008; 17(1).

[15] Ansari NN, Naghdi S, Hasson S, Valizadeh L, Jalaie S. Validation of a Mini-Mental State Examination (MMSE) for the Persian population: A pilot study. Applied Neuropsychology. 2010; 17(3):190-5. [DOI:10.1080/09084282.2010.499773] [PMID]

[16] Beck AT, Steer RA, Brown GK. Beck depression inventory-II. San Antonio: Psychological Corporation; 1996.

[17] Ghassemzadeh H, Mojtabai R, Karamghadiri N, Ebrahimkhani N. Psychometric properties of a Persian-language version of the Beck Depression Inventory-Second edition: BDI-II-PERSIAN. Depression and Anxiety. 2005; 21(4):185-92. [DOI:10.1002/ da.20070] [PMID]

[18] Moghaddam JF, Nakhaee N, Sheibani V, Garrusi B, Amirkafi A. Reliability and validity of the Persian version of the Pittsburgh Sleep Quality Index (PSQI-P). Sleep and Breathing. 2012; 16(1):79-82. [DOI:10.1007/s11325-010-0478-5] [PMID]

[19] Asghari A, Saed F, Dibajnia P. Psychometric properties of the Depression Anxiety Stress Scales-21 (DASS-21) in a non-clinical Iranian sample. International Journal of Psychology. 2008; 2(2):82-102.
[20] Lovibond S, Lovibond P. Manual for the Depression Anxiety Stress Scales. Sidney: Psychology Foundation of Australia; 1995.

[21] Morris S, Morris ME, Iansek R. Reliability of measurements obtained with the Timed "Up \& Go" test in people with Parkinson disease. Physical Therapy. 2001; 81(2):810-8. [DOI:10.1093/ ptj/81.2.810] [PMID]

[22] Frazzitta G, Maestri R, Ferrazzoli D, Riboldazzi G, Bera R, Fontanesi $\mathrm{C}$, et al. Multidisciplinary intensive rehabilitation treatment improves sleep quality in Parkinson's disease. Journal of Clinical Movement Disorders. 2015; 2(1):11. [DOI:10.1186/ s40734-015-0020-9] [PMID] [PMCID]

[23] Salavati M, Negahban H, Mazaheri M, Soleimanifar M, Hadadi M, Sefiddashti L, et al. The Persian version of the Berg Balance Scale: Inter and intra-rater reliability and construct validity in elderly adults. Disability and Rehabilitation. 2012; 34(20):16958. [DOI:10.3109/09638288.2012.660604]

[24] Asgari T, Hadian MR, Nakhostin-Ansari N, Abdolvahhab M, Jalili M, Faghih-Zadeh S. [Berg Balance Scale reliability for evaluation in children with spastic diplegia (Persian)]. Archive of Rehabilitation. 2007; 8(2):13-6.

[25] Duncan PW, Weiner DK, Chandler J, Studenski S. Functional reach: A new clinical measure of balance. Journal of Gerontology. 1990; 45(6):M192-7. [DOI:10.1093/geronj/45.6.M192]

[26] Butland RJ, Pang JA, Gross ER, Woodcock AA, Geddes DM. Two-, six-, and 12-minute walking tests in respiratory disease. British Medical Journal (Clinical Research ed.). 1982; 284(6329):1607-8. [DOI:10.1136/bmj.284.6329.1607]

[27] Connelly DM, Thomas BK, Cliffe SJ, Perry WM, Smith RE. Clinical utility of the 2-minute walk test for older adults living in long-term care. Physiotherapy Canada. 2009; 61(2):78-87. [DOI:10.3138/physio.61.2.78]

[28] Severiano MIR, Zeigelboim BS, Teive HAG, Santos GJB, Fonseca VR. Effect of virtual reality in Parkinson's disease: A prospective observational study. Archives of neuro-psychiatry. 2018; 76(2):78-84. [DOI:10.1590/0004-282x20170195] [PMID]

[29] Petzinger GM, Holschneider D, Fisher B, McEwen S, Kintz $\mathrm{N}$, Halliday M, et al. The effects of exercise on dopamine neurotransmission in Parkinson's disease: Targeting neuroplasticity to modulate basal ganglia circuitry. Brain Plasticity. 2015; 1(1):2939. [DOI:10.3233/BPL-150021] [PMID] [PMCID]

[30] Lauterbach EC. The neuropsychiatry of Parkinson's disease and related disorders. Psychiatric Clinics. 2004; 27(4):801-25. [DOI:10.1016/j.psc.2004.07.001]

[31] Schor CM. Neuromuscular plasticity and rehabilitation of the ocular near response. Optometry and Vision Science. 2009; 86(7):E788-E802. [DOI:10.1097/OPX.0b013e3181ae00a5] [PMID]

[32] Badaracco C, Labini FS, Meli A, Tufarelli D. Oscillopsia in labyrinthine defective patients: Comparison of objective and subjective measures. American Journal of Otolaryngology. 2010; 31(6):399-403. [DOI:10.1016/j.amjoto.2009.06.002] [PMID]

[33] Cromwell RL, Newton RA, Carlton LG. Horizontal plane head stabilization during locomotor tasks. Journal of Motor Behavior. 2001; 33(1):49-58. [DOI:10.1080/00222890109601902] [PMID] 